\title{
Determinantes sociais da saúde que impactam a vivência da imigração no Brasil
}

\author{
Social determinants of health that impact the experience of immigration in Brazil \\ Determinantes sociales de la salud que impactan la experiencia de la inmigración en Brasil
}

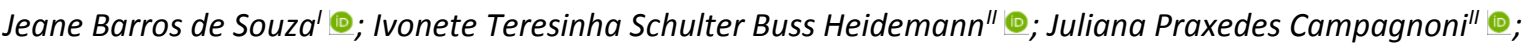

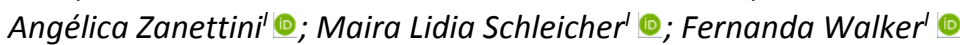

'Universidade Federal da Fronteira do Sul, Chapecó, SC, Brasil; Universidade Federal de Santa Catarina, Florianópolis, SC, Brasil

\begin{abstract}
RESUMO
Objetivo: compreender as percepções dos imigrantes haitianos sobre os Determinantes Sociais da Saúde que impactam a vivência da imigração. Método: pesquisa qualitativa, do tipo pesquisa ação participante, fundamentada no Itinerário de Pesquisa de Paulo Freire, que constitui três fases: Investigação Temática; Codificação e Descodificação; Desvelamento Crítico. Realizou-se Círculo de Cultura, no segundo semestre de 2019, com a participação de 12 imigrantes haitianos, estudantes universitários, residentes no oeste de Santa Catarina, Brasil, após aprovação pelo Comitê de Ética em pesquisa. Resultados: nos diálogos emergiram duas temáticas para discussão como determinação social da saúde e doença: saúde do imigrante no Brasil; desafios de estudar e trabalhar. Conclusão: A iniquidade de acesso aos direitos, escasso tempo para dormir e praticar exercícios físicos, saudade do Haiti, dificuldade financeira, adaptação à cultura brasileira e discriminação foram apontados como fatores determinantes que afetam a saúde. Urge a necessidade da construção de políticas públicas que garantam os direitos dos imigrantes no Brasil.
\end{abstract}

Descritores: Enfermagem em Saúde Pública; Determinantes Sociais da Saúde; Emigrantes e Imigrantes; Vulnerabilidade em Saúde.

\begin{abstract}
Objective: to understand the perceptions of Haitian immigrants about the social determinants of health that impact on the experience of immigration. Method: this qualitative, participant action study was based on Paulo Freire's research itinerary, which consists of three phases: thematic research; encoding and decoding; and critical unveiling. A "culture circle" was held, after ethics committee approval, in the second half of 2019, with the participation of 12 immigrant Haitian university students residing in western Santa Catarina, Brazil. Results: in the dialogues, two themes emerged for discussion as representing the social determination of health and disease: immigrants' health in Brazil; and challenges of studying and working. Conclusion: inequity in access to rights, lack of time to sleep and exercise, homesickness for Haiti, financial difficulties, adaptation to Brazilian culture, and discrimination were identified as determinant factors that affect health. There is an urgent need to build public policies to guarantee immigrants' rights in Brazil.
\end{abstract}

Descriptors: Public Health Nursing; Social Determinants of Health; Emigrants and Immigrants; Health Vulnerability.

\section{RESUMEN}

Objetivo: comprender las percepciones de los inmigrantes haitianos sobre los determinantes sociales de la salud que impactan en la experiencia de la inmigración. Método: este estudio cualitativo de acción participante se basó en el itinerario de investigación de Paulo Freire, que consta de tres fases: investigación temática; codificación y decodificación; y revelación crítica. Se realizó un "círculo cultural", luego de la aprobación del comité de ética, en el segundo semestre de 2019, con la participación de 12 estudiantes universitarios haitianos inmigrantes residentes en el occidente de Santa Catarina, Brasil. Resultados: en los diálogos surgieron dos temas de discusión que representan la determinación social de la salud y la enfermedad: la salud de los inmigrantes en Brasil; y desafíos de estudiar y trabajar. Conclusión: la inequidad en el acceso a los derechos, la falta de tiempo para dormir y hacer ejercicio, la nostalgia por Haití, las dificultades económicas, la adaptación a la cultura brasileña y la discriminación fueron identificadas como factores determinantes que afectan la salud. Urge construir políticas públicas para garantizar los derechos de los inmigrantes en Brasil.

Descriptores: Enfermería de Salud Pública; Determinantes Sociales de la Salud; Emigrantes e Inmigrantes; Vulnerabilidad em Salud.

\section{INTRODUÇÃO}

A imigração contemporânea é global, sendo que o Brasil tem recebido imigrantes de várias nações e por razões heterogêneas ${ }^{1}$. Ao observar os processos migratórios internacionais ao Brasil, evidencia-se que os haitianos ganharam destaque pelo volume de deslocamentos em busca de trabalho, longe das consequências de terremotos ${ }^{2}$. Além disso, há o acordo entre governos haitiano e brasileiro, mediado pela Organização das Nações Unidas (ONU), que facilitou a integração desses imigrantes pelo fornecimento do registro no cadastro nacional de pessoas físicas, permitindo inclusão ágil no mercado de trabalho³.

Os imigrantes constituem um público vulnerável a diversos Determinantes Sociais da Saúde (DSS), definidos como fatores que influenciam, afetam e/ou determinam a saúde das pessoas. Salienta-se que o equilíbrio do processo saúde-

Agradecimentos à Coordenação de Aperfeiçoamento de Pessoal de Nível Superior (CAPES) - Programa Nacional de Pós-Doutorado - Processo 88887.357993/2019-00. Autora correspondente: Angélica Zanettini. E-mail: angeliica.zanettini@gmail.com

Editora responsável: Mercedes Neto 
doença é determinado por múltiplos fatores de origem social, econômica, cultural, ambiental e biológica. Porém, apesar dos fatores externos ao indivíduo serem conhecidos internacionalmente, nem sempre foram inseridos ao formularemse políticas de saúde ${ }^{4}$, necessitando compreendê-los para criar estratégias de intervenção adequadas à realidade, em prol da qualidade de vida ${ }^{5}$.

Nesse cenário, torna-se premente a realização de pesquisas que possibilitam conhecer a realidade dos imigrantes e que contribuam na elaboração de políticas e práticas que desvelam os DSS que envolvem esse público. Por conseguinte, enfermeiros e demais profissionais da saúde poderão intervir positivamente nos DSS, em busca de diminuir vulnerabilidades dessa população, justificando a relevância desse estudo.

Assim, despontou a questão norteadora: quais as percepções dos imigrantes haitianos sobre os DSS que impactam a vivência da imigração? Logo, o estudo objetivou compreender as percepções dos imigrantes haitianos sobre os DSS que impactam a vivência da imigração.

\section{REFERENCIAL TEÓRICO}

O estudo referenciou-se nos pressupostos teórico-metodológicos de Paulo Freire, considerando que todo indivíduo possui saberes que devem ser valorizados, com incentivo da autonomia, instigando o conhecimento e empoderamento pela práxis dialógica, que ocorre no Círculo de Cultura ${ }^{6,7}$.

O Círculo de Cultura é um encontro de pessoas que discutem temas comuns pelo diálogo, em relações horizontalizadas e reflexivas, partilhando experiências. É mediado por um facilitador que problematiza os temas emergentes, visando instigar o conhecimento coletivo, em que todos os participantes se enriquecem e transformam ${ }^{7,8}$. Nesse espaço, emergem possibilidades para pesquisadores e enfermeiros estabelecerem vínculos, em prol da saúde e empoderamento dos imigrantes.

\section{MÉTODO}

Pesquisa qualitativa, tipo ação participante ${ }^{9}$, em que o estudo resulta em uma ação prática e concreta, considerando o pesquisar um processo pedagógico interligado à transformação social ${ }^{8}$. Fundamentou-se no Itinerário de Pesquisa Freireano, que possui três fases: 1) Investigação Temática: momento de identificar os temas geradores; 2 ) Codificação e Descodificação: elaboração dos códigos que estimulam a reflexão crítica sobre a situação vivida, refletindo acerca da superação de situações limite; 3) Desvelamento Crítico: socialização e consolidação das ideias sob a reflexão crítica da realidade, proporcionando a compreensão de como atuar para transformar ${ }^{6,7}$.

Para organizar o Círculo de Cultura, encaminhou-se um e-mail para os 91 estudantes haitianos de uma universidade pública do oeste catarinense, convidando-os para participar de uma reunião. Compareceram 22 estudantes de diferentes períodos, conciliando horário com 12 deles, que assim tornaram-se os participantes do estudo. Como critérios de inclusão foram considerados imigrantes haitianos acima de 18 anos e estudantes universitários. Como critérios de exclusão aqueles que não frequentavam regularmente as aulas.

O Círculo durou duas horas, numa sala de aula da universidade, no segundo semestre de 2019. Contou-se com o apoio de gravador de áudio. Para tornar a discussão sobre DSS concreta e interativa, optou-se em percorrer as etapas do Itinerário por meio de uma analogia com a construção simbólica de uma árvore, oportunizando realizar todas as fases do Itinerário em um único encontro. Uma árvore de papel foi construída e exposta ao chão, cujas peças foram montadas durante o encontro.

Para a Investigação Temática (coleta de dados), a mediadora instigou o diálogo com o questionamento: o que impacta a saúde na vivência da imigração no Brasil? Cada participante recebeu um papel, representando as raízes da árvore, sendo convidados a responder o questionamento por meio de uma palavra. Os imigrantes compartilharam suas percepções com o grupo, expondo as raízes da árvore ao chão. Após amplo debate, definiram dois temas geradores para discutir no Círculo: saúde do imigrante no Brasil; desafios de estudar e trabalhar.

Na etapa da Codificação e Descodificação (coleta e análise de dados), o caule da árvore foi montado e dividido em duas partes. Na primeira abordou-se sobre os DSS que envolviam a saúde dos imigrantes haitianos, por meio da pergunta: como é a saúde do imigrante no Brasil? A segunda parte do caule foi destinada para debater sobre os desafios de ser estudante e trabalhador, com a questão: como é ser um imigrante estudante e trabalhador?

No Desvelamento Crítico (análise de dados), os participantes inseriram as folhas da árvore, refletindo sobre qual seria o DSS com maior impacto em suas vidas e como pretendiam promover sua saúde. Todos se retroalimentaram das reflexões coletivas, despontando novos conhecimentos e possibilidades.

Os diálogos do Círculo de Cultura foram transcritos e organizados em pastas digitais, conforme os dois temas. Para análise dos dados realizou-se leitura criteriosa dessas informações registradas nas pastas. Tal análise realizou-se 
concomitantemente ao desenvolvimento do Círculo, como prevê o Itinerário freireano ${ }^{6}$, em que os participantes expressaram as concepções relacionadas aos temas, tornando-os mais visíveis.

A pesquisa seguiu os preceitos éticos da Resolução 466 de 2012, destacando que os imigrantes assinaram o Termo de Consentimento Livre e Esclarecido. Para garantir o anonimato, foram denominados por nome de árvores. A pesquisa foi aprovada pelo Comitê de Ética em Pesquisa da Universidade Federal da Fronteira Sul, em 14 de maio de 2019, parecer número 3.324.430.

\section{RESULTADOS}

Os 12 participantes tinham idade entre 21 e 25 anos, sendo nove do sexo masculino e três do sexo feminino, residentes entre cinco meses à seis anos no Brasil. Quanto aos cursos universitários: um acadêmico de Enfermagem, três de Pedagogia, dois de Engenharia Ambiental, dois de Geografia, dois de Matemática e dois de Letras.

\section{Saúde do imigrante no Brasil}

Os participantes apontaram as dificuldades encontradas como determinação social do processo saúde-doença na vivência da imigração, desvelando o desafio em manter uma alimentação saudável, em virtude das questões econômicas:

No mercado tudo que é saudável é caro. Eu compro o que o meu dinheiro pode comprar, daí fica difícil ter uma alimentação saudável. (Bambu)

Os participantes também destacaram a falta de tempo para prática de exercícios físicos para manter uma vida mais saudável:

Eu gosto de me exercitar, mas me falta tempo. (Jatobá)

Os haitianos referiram ter dificuldades para estabelecer amizades com os brasileiros, sentindo falta da família, o que gera tristeza:

Aqui a gente tem poucos amigos, então, a saudade da família fica maior ainda. Isso me dá tristeza. (Figueira)

Foi dialogado sobre o desafio do aprendizado da língua portuguesa:

Adaptação no início não é fácil... um desafio é o português, a língua é difícil. Ficar sem saber nada do que estão falando a sua volta não é legal. (Castanheira)

Outra questão levantada nos diálogos foi a falta que sentem da cultura haitiana e das diferentes maneiras de adorar a Deus na igreja:

Eu sinto falta da cultura do Haiti, da maneira que vivemos lá. (Juazeiro)

Aqui muita coisa é diferente, até na igreja, eles fazem pouco barulho. Parecemos estranhos para eles. (Bambu)

Alguns haitianos revelaram frustração com os brasileiros, que pouco ou nenhum interesse demonstram em conhecer seu país e suas histórias de vida. Também apontaram que a falta de informações gera iniquidade na vivência da imigração:

Aqui parece que as pessoas não têm curiosidade de saber sobre o Haiti e sobre nossas vidas. A gente precisa saber tudo sobre o Brasil, mas os brasileiros geralmente não querem saber nada de nós. (Seringueira)

No Brasil tem leis diferentes e a gente não conhece, não sabemos sobre os nossos direitos aqui. Daí, acaba faltando equidade porque em muitos lugares, não somos tratados igual aos brasileiros. (Oliveira)

\section{Desafios de estudar e trabalhar}

Os imigrantes haitianos dialogaram que ao chegar ao Brasil encontraram oportunidades para estudar em universidades, mas depararam-se com a falta de apoio dos docentes nesse processo, enfrentando o preconceito:

Falta o professor trabalhar a inclusão, tem que entender que na sala não tem só brasileiro. Tem imigrantes que às vezes acabaram de chegar no Brasil e não falam bem o português. Falta apoio dos professores. (Pinus)

Teve uma menina que tirou nota 7,5. O professor parabenizou essa aluna. E o meu amigo, que é haitiano, tirou nota 10 e não recebeu parabéns. Isso é preconceito. (Bambu)

Para os imigrantes haitianos, a oportunidade de cursar o ensino superior é a concretização de um sonho. Entretanto, para que esse sonho se torne possível, por vezes se faz necessário estudar e trabalhar, o que dificulta a permanência nos estudos:

Já teve imigrante haitiano que tentou fazer um curso integral e trabalhando a noite, mas não dormia e acabou ficando doente e desistindo de estudar. (Jatobá)

Mas as dificuldades vivenciadas e excesso de responsabilidades comprometem a saúde:

Nós temos muita coisa para fazer na universidade e no trabalho. Não temos tempo para descansar e dormir uma noite inteira e acabamos adoecendo. (Juazeiro) 
Quando questionados sobre o trabalho, constatou-se que a maioria dos entrevistados encontraram oportunidades laborais apenas nas empresas frigoríficas, sofrendo discriminação por ser estrangeiro e negro:

Por eu ser estrangeiro e também por eu ser negro, se recusam em me dar um bom trabalho, isso é discriminação e preconceito. (Cedro)

Muitos trabalhadores imigrantes são discriminados, sem as mesmas oportunidades dos brasileiros. (Juazeiro)

Ao findar o Círculo de Cultura, desvelou-se que os imigrantes saíram transformados, trocando experiências em busca das possibilidades para promover seu bem-estar e autocuidado, com gratidão pelos momentos vivenciados, evidenciando empoderamento e desejo de que os resultados tragam aprimoramento e novas reflexões sobre a saúde dos imigrantes:

Eu quero agradecer porque aprendi muito. Vou sair diferente de como entrei. (Juazeiro)

Eu espero que a gente cuide mais da nossa saúde, que possamos nos empoderar, buscar nossos direitos. Que essa pesquisa traga muitos resultados porque alguém precisa ver isso e vocês estão se preocupando com a saúde dos imigrantes. (Bambu)

\section{DISCUSSÃO}

São vários DSS que impactam a vivência da imigração. O ser humano tem direitos, sendo que um deles envolve a alimentação saudável. Contudo, na contemporaneidade os alimentos são distribuídos de acordo com a lei da oferta e da demanda ${ }^{10}$. A inatividade física é outro fator que está diretamente ligada a diminuição da qualidade de vida, ao aumento do número de doenças, a obesidade e a mortalidade. A realização de atividades físicas e ou de lazer são subsídios para prevenção e promoção da saúde, resultando em uma melhora na percepção da saúde ${ }^{11}$.

Quanto a saúde mental, cabe salientar que durante o processo de assentamento, a falta de amizades pode desencadear o isolamento ${ }^{12}$. Somado a isso, dominar a língua apresenta-se como uma necessidade e não uma escolha, pois têm impacto recorrente no viver dos imigrantes na busca por oportunidades de trabalho, educação, saúde, novas amizades, entre outros ${ }^{13}$

A participação em uma igreja, mantendo a religiosidade do Haiti, é uma função notável no sentido de manter a saúde espiritual, tendo ligação simbólica com o país de origem. No entanto, há um impacto diante da ruptura das raízes familiares e das dificuldades da inserção em uma nova cultura, como evidenciado pelo estranhamento na igreja, o que pode provocar o isolamento, o desânimo e a saudade ${ }^{14}$. Esse confronto de valores que subsidiam as identidades tanto da sociedade que acolhe quanto dos imigrantes, pode gerar relações dificultosas devido a diferenciação entre nativos e imigrantes ${ }^{15}$.

A Lei de Migração visa estabelecer alguns princípios e garantias básicas aos migrantes, bem como o direito de regularização documental, uma acolhida humanitária, a inclusão tanto social como laboral ${ }^{16}$. Desse modo, denota-se que permitir a entrada dos haitianos no Brasil não é suficiente, fazendo-se necessário aperfeiçoar e fiscalizar o cumprimento da legislação brasileira para que os direitos à cidadania sejam atendidos, buscando a disseminação de informações, a fim de que se empoderem dos seus direitos e deveres para recomeçar suas vidas no novo país.

A vulnerabilidade está presente na vida dos imigrantes e para alcançar a igualdade dos diferentes, é necessário ofertar-lhes proteção especial, ou seja, a constituição brasileira vigente é extremamente cuidadosa ao referir que os direitos são inatos a todo indivíduo, não importando se são nacionais ou estrangeiros ${ }^{1}$. Somado a isso, o Sistema Único de Saúde (SUS) do Brasil defende o princípio da equidade aos cidadãos do território brasileiro ${ }^{17}$. Portanto, antes de ser um estrangeiro, os imigrantes são indivíduos que possuem direitos essenciais, que inclui a educação.

Nesse cenário educacional desponta a relevância do apoio docente para auxiliar os estudantes em suas dificuldades, incentivando-os a prosseguir nos estudos. Entende-se o espaço universitário como um local apropriado para discutir sobre preconceito e discriminação, mas não como um espaço que promove tais situações. Além disso, deve-se pensar na educação inclusiva, que apesar de ser discutida em diversos setores, ainda falta maior aplicação no contexto universitário quanto ao reconhecimento e atendimento das necessidades de cada estudante ${ }^{15}$. Para efetivar a inclusão é imprescindível formular mecanismos de auxílio que criem condições reais de permanência, pois sem apoio institucional pode haver processos de exclusão nas universidades.

Sabe-se que existem políticas de permanência que são oferecidas pelo governo brasileiro, mas que não conseguem alcançar todos os estudantes universitários, o que dificulta a permanência e excelência, uma vez que muitos são obrigados a trabalhar para conseguir estudar ${ }^{18}$, prejudicando na manutenção de um sono adequado. Observa-se uma significativa diminuição do tempo e qualidade do sono da população, com repercussões sobre a função psicológica, sistema imunológico, desempenho e humor. Assim, garantir qualidade no sono é relevante para a manutenção de uma vida saudável ${ }^{19}$.

Os imigrantes exercem atividades laborais que exigem pouco grau de instrução e que demandam esforço e carga horária elevada, com baixos salários ${ }^{20}$, emergindo a discriminação e o preconceito. Na discriminação racial as pessoas que não fazem parte de grupos raciais dominantes sofrem distinções em virtude de sua cor da pele, o que resulta em 
desigualdade de vários recursos: oportunidades educacionais e profissionais, rendimentos e mobilidade social. $\mathrm{O}$ preconceito está relacionado com questões afetivas, diante da preferência por um grupo em detrimento de outro, enquanto a discriminação consiste em um comportamento ${ }^{21}$.

Nesse sentido, os eventos estressores ligados ao processo imigratório e de aculturação, somados a presença de discriminação e preconceito impactam fortemente na qualidade de vida dos imigrantes, influenciando a determinação social do processo saúde-doença ${ }^{5}$. É essencial que grupos que sofrem processos de exclusão, como os imigrantes haitianos, possam verbalizar suas experiências de vida, manifestar suas necessidades e incitar esferas da sociedade mediante estratégias de enfrentamento e superação de suas condições de vulnerabilidade ${ }^{22}$.

Sugere-se a realização de outros estudos que versem sobre as condições de vida dos imigrantes, dando-lhes voz ativa, assim como ocorreu no processo desse estudo, que trouxe contribuições para a área da Enfermagem, Saúde Coletiva e Educação, instigando profissionais e sociedade a se mobilizarem para atender essa clientela conforme suas reais necessidades, dando visibilidade a essa população que tanto carece de informações e garantia de direitos, em prol da qualidade de vida.

Como limitação do estudo cita-se a inserção de apenas imigrantes haitianos na pesquisa. Incentiva-se a realização de estudos com outras nacionalidades, a fim de conhecer os impactos dos DSS no contexto imigratório, em busca de aprimoramento de políticas no setor Saúde, Educação e Trabalho no Brasil.

\section{CONSIDERAÇÕES FINAIS}

Os DSS percebidos pelos imigrantes haitianos, que impactam a vivência da imigração, envolvem a dificuldade de consumir alimentos considerados saudáveis por serem de valor monetário elevado, saudade da família e cultura haitiana, escassas condições financeiras, falta de equidade e dificuldade de se comunicar com os brasileiros. Destacouse ainda a falta de tempo para dormir e praticar exercícios físicos.

Também foi reforçado a presença do preconceito e a discriminação no trabalho, no convívio social e na universidade, o que dificulta a inserção e adaptação no Brasil. Os reflexos da escravidão, ainda visíveis nos atos de exploração, preconceito e discriminação com os imigrantes, necessitam ser combatidos em suas amplas formas. Assim, ações que reafirmam a igualdade na diversidade por meio de um diálogo intercultural são urgentes, salientando-se a relevância do papel do professor como fonte de apoio para superar as barreiras na universidade.

Nesse âmbito, urge o engajamento dos órgãos públicos e da sociedade civil na construção de políticas públicas que garantam os direitos dos imigrantes para que possam viver uma vida digna no país ao qual está inserido. Ainda, que a enfermagem em saúde púbica e demais profissionais da área possam voltar os olhos para este público, que tanto carece de maior visibilidade e cuidado.

\section{REFERÊNCIAS}

1. Lima SS, Silva LMM. The immigrants in Brazil, its vulnerability and the principle of equality. RBPP [internet]. 2017 [cited 2019 Dec 10]; 7(2):384-403. DOI: https://doi.org/10.5102/rbpp.v7i2.4804.

2. Fernandes D. (Coord.). Relatório do Projeto 'Estudos sobre a Migração Haitiana ao Brasil e Diálogo Bilateral'. Organização Internacional para as Migrações - OIM. Belo Horizonte: TEM/IOM-OIM/PUC Minas/Gedep, 2014 [cited 2020 Jan 07]; Available from: http://obs.org.br/cooperacao/download/34_7a099729afe2d4aaf109503e6daf3908.

3. Granada D, Carreno I, Ramos N, Ramos MCP. Debating health and migrations in a context of intense human mobility. Interface (Botucatu) [Internet]. 2017 Jun [cited 2020 Jan 07]; 21(61):285-96. DOI: https://doi.org/10.1590/1807-57622016.0626.

4. Carrapato P, Correia P, Garcia B. Health determinants in Brasil: searching for health equity. Saude soc. [Internet]. 2017 [cited 2020 Jan 07]; 26(3):676-89. DOI: https://doi.org/10.1590/s0104-12902017170304.

5. Becker APS, Borges L. Psychosocial dimensions of migration for the family. Bol. Acad. Paul. Psicol. [Internet]. 2015 [cited 2020 Feb 19]; 35(88):124-44. Available from: http://pepsic.bvsalud.org/scielo.php?script=sci_arttext\&pid=S1415$711 X 2015000100009 \& \operatorname{lng}=p t \& n r m=i s o$.

6. Heidemann ITSB, Dalmolin IS, Rumor PCF, Cypriano CCC, Costa MFBNA, Durand MK. Reflections on Paulo Freire's research itinerary: contributions to health. Texto \& contexto enferm. [Internet]. 2017 [cited 2019 Nov 20]; 26(4):e0680017. DOI: http://dx.doi.org/10.1590/0104-07072017000680017.

7. Freire P. Pedagogia do Oprimido. Rio de Janeiro (RJ): Paz e Terra; 2019.

8. Antonini FO, Heideman ITSB. Paulo Freire's research itinerary: contributions for promoting health in the teaching profession. Rev. Bras. Enferm. [Internet]. 2020 [cited 2020 Nov 20]; 73(4):20190164. DOI: https://doi.org/10.1590/0034-7167-2019-0164.

9. Felcher CDO, Ferreira ALA, Folmer V. Da pesquisa-ação à pesquisa participante: discussões a partir de uma investigação desenvolvida no facebook. EENCI [Internet]. 2017 [cited 2019 Nov 20]; 12(7):1-18. Available from: https://if.ufmt.br/eenci/artigos/Artigo_ID419/v12_n7_a2017.pdf.

10. Guerra LDS, Cervato-Mancuso AM, Bezerra ACD. Food: a disputed human right - a thematic focus for comprehension and action in food and nutritional security. Ciênc. Saúde Colet. [Internet]. 2019 [cited 2020 Feb 3]; 24(9):3369-94. DOI:

http://dx.doi.org/10.1590/1413-81232018249.20302017. 
11. Fritsch F, Dudel-Mayer B, Ubessi L, Kirchner R, Barbosa D, Fernandes-Stumm E. Physical activity, leisure and evaluation of health in the perspective of users in hemodialysis. Rev. Pesqui. [Internet]. 2015 [cited 2020 Feb 21]; 7(4):3263-73. DOI: https://doi.org/10.1016/j.healthplace.2015.03.016.

12. Souza JB, Heidemann ITSB, Walker F, Schleicher ML, Campagnoni JP. Reflections on health with Haitian immigrants through Paulo Freire's Research Itinerary. Rev. Eletr. Enferm. [Internet]. 2020 [cited 2020 Nov 26]; 22:60792. Available from: https://revistas.ufg.br/fen/article/download/60792/35679/.

13. Balestro AC, Pereira T. Language and culture in the feminization of migration in Brazil. Trab. linguist. apl. [Internet] 2019 [cited 2020 Feb 15]; 58(2) 779-94. DOI: https://doi.org/10.1590/010318138654049455422.

14. Tomelin KN, Dias APL, Sanchez CNM, Peres J. Inclusive approach on higher education: challenges and experiences of a nucleus for faculty and student support. Rev. psicopedag. [Internet]. 2018 [cited 2020 Jan 05]; 35(106):94-103. Available from: http://pepsic.bvsalud.org/scielo.php?script=sci_arttext\&pid=S0103-84862018000100011\&lng=pt\&tlng=pt.

15. Mejía MRG, Cazarotto RT. The role of immigrant women in the transnational family which mobilizes haitian migration in Brazil. Repocs [Internet]. 2017 [cited 2019 Dec 03]; 14(27):171-90. Available from: http://www.periodicoseletronicos.ufma.br/index.php/rpcsoc/article/view/6452.

16. Brasil. Lei no 13.445 de 24 maio de 2017. Institui Lei de Migração. Diário Oficial da União. Brasília (DF): Poder Legislativo; 2017 [cited 2019 Dec 03]; Available from: http://pesquisa.in.gov.br/imprensa/jsp/visualiza/index.jsp?data=25/05/2017\&jornal=1\&pagina=1\&totalArquivos=304.

17. Barros FPC, Sousa MF. Equity: concepts, meanings and implications for the Brazilian National Health System. Saude soc. [Internet]. 2016 [cited 2020 Jan 04]; 25(1):9-18. DOI: https://doi.org/10.1590/S0104-12902016146195.

18. Wittkowski JRT, Da Costa SP, Meneghel SM, Martins RM. The top education teacher in inclusion and affirmative action policies. Braz. J. Dev. [Internet] 2019 [cited 2020 Jan 09]; 5(9):13789-805. DOI: https://doi.org/10.34117/bjdv5n9-012.

19. Marques BS, Silva MAC. Trabalhadores-alunos: motivações e desafios que configuram um cenário de luta. In: Anais do I Encontro Internacional de Gestão, Desenvolvimento e Inovação. 2017 set 12-14; Naviraí, Brasil. Naviraí (MS): Núcleo de Pesquisa em “Gestão, Desenvolvimento e Inovação" (NGDI) - UFMS Universidade Federal de Mato Grosso do Sul; 2017. p. 1-16. [cited 2019 Dec 03]; Available from: https://periodicos.ufms.br/index.php/EIGEDIN/article/view/4337.

20. Neves GSML, Macedo P, Gomes MM. Sleep disorders: up to date. Rev. Bras. Neurol. [Internet]. 2017 [cited 2020 Feb 18]; 53(3):19-30. Available from: http://docs.bvsalud.org/biblioref/2017/12/876873/rbn-533-3-transtornos-do-sono-1-2.pdf.

21. Daflon VT, Carvalhaes F, Júnior JF. Deeper than Skin: Browns' and Blacks' Perceptions of Discrimination in Brazil [Internet]. 2017 [cited 2020 Jan 19]; 60(2):293-330. DOI: https://doi.org/10.1590/001152582017121

22. Freitas MA, de Mattos ATR, Gomes WZ, Caccia-Bava MCGG. Who are they, what do they talk about and who listens to the poor? Ciênc. saúde colet. [Internet]. 2017 Dez [cited 2020 Jan 27]; 22(12):3859-82. DOI: http://dx.doi.org/10.1590/1413812320172212.24852017 